\title{
Hybrid Fitness in a Locally Adapted Parasite
}

\author{
Mark F. Dybdahl, ${ }^{1, *}$ Jukka Jokela, ${ }^{2}$ Lynda F. Delph, ${ }^{3}$ Britt Koskella, ${ }^{3}$ and Curtis M. Lively ${ }^{3}$
}

1. School of Biological Sciences, Washington State University, Pullman, Washington 99164;

2. EAWAG, Swiss Federal Institute of Aquatic Sciences and Technology, Überlandstrasse 133, CH-8600 Dübendorf, Switzerland; and Swiss Federal Institute of Technology Zürich, Institute of Integrative Biology, Universitätstrasse 16, 8092 Zürich, Switzerland;

3. Department of Biology, Indiana University, Bloomington, Indiana 47405

Submitted January 25, 2008; Accepted June 27, 2008;

Electronically published October 24, 2008

Online enhancements: appendix tables and figure.

Aвstract: The parasite (Red Queen) hypothesis for the maintenance of sexual reproduction and genetic diversity assumes that hostparasite interactions result from tight genetic specificity. Hence, hybridization between divergent parasite populations would be expected to disrupt adaptive gene combinations, leading to reduced infectivity on exposure to parental sympatric hosts, as long as gene effects are nonadditive. In contrast, hybridization would not cause reduced infectivity on allopatric hosts unless the divergent parasite populations possess alleles that are intrinsically incompatible when they are combined. In three different experiments, we compared the infectivity of locally adapted parasite (trematode) populations with that of $F_{1}$ hybrid parasites when exposed to host (snail) populations that were sympatric to one of the two parasite populations. We tested for intrinsic genetic incompatibilities in two experiments by including one host population that was allopatric to both parasite populations. As predicted, when the target host populations were sympatric to the parasite populations, the hybrids were significantly less infective than the parental average, while hybrid parasites on allopatric hosts were not, thereby ruling out intrinsic genetic incompatibilities. The results are consistent with nonadditive gene effects and tightly specific host-driven selection underlying parasite divergence, as envisioned by coevolutionary theory and the Red Queen hypothesis.

Keywords: host-parasite interactions, hybrid fitness, sexual reproduction, Red Queen hypothesis, dominance, epistasis.

* Corresponding author; e-mail: dybdahl@wsu.edu.

Am. Nat. 2008. Vol. 172, pp. 772-782. () 2008 by The University of Chicago. 0003-0147/2008/17206-50207\$15.00. All rights reserved.

DOI: $10.1086 / 592866$
The parasite (or Red Queen) hypothesis for the maintenance of sex is predicated on the idea that different parasite genotypes are limited to infecting a different subset of host genotypes. As a consequence, parasites are expected to impose time-lagged selection against common host genotypes, leading to the maintenance of local genetic diversity in host populations and possibly to selection for cross-fertilization among sexual hosts (Jaenike 1978; Hamilton 1980; Howard and Lively 1994; Peters and Lively 1999; Otto and Nuismer 2004; Peters and Lively 2007). As a further consequence of genetic specificity, hostparasite coevolution within geographically structured populations is expected to produce local adaptive divergence among parasite populations (Gandon et al. 1996; Lively 1999; Nuismer 2006).

Several empirical studies have demonstrated genetic specificity for infection in different host-parasite genetic interactions (Lively and Dybdahl 2000; Carius et al. 2001). For example, Carius et al. (2001) showed that no single Daphnia clone was resistant to all parasite strains and, similarly, that no parasite strain was infective to all Daphnia clones. Here we take a different approach to understanding genetic specificity. We compare the fitness of interpopulation hybrid parasites with the fitnesses of their locally adapted parental populations. The comparison of hybrids and their parental populations is useful for determining the genetic basis of divergence between populations (Hatfield and Schluter 1999; Hines et al. 2004) and for inferring the genetic basis of specific traits (Lynch and Walsh 1998; Fritz et al. 1999, 2003). We were interested in whether outbreeding depression existed among locally adapted parasite populations and its genetic causes.

We compared the fitness of $F_{1}$ hybrids between locally adapted parasite populations (the trematode Microphallus sp.) with the fitness of their parental populations, and we made these comparisons on both sympatric and allopatric hosts (the freshwater snail Potamopyrgus antipodarum). The snail host is native to freshwater habitats of New Zealand, where sexual and asexual individuals coexist in many populations (Lively 1987; Dybdahl and Lively 1995). The trematode is the most common parasite infecting the 
snail, reaching levels of infection in excess of 50\% in many locations (Jokela and Lively 1995; Lively and Jokela 2002). The infection rate is also positively correlated with the frequency of sexual P. antipodarum, suggesting that these parasites may be a factor selecting for sexual reproduction in their host (Lively 1987, 1992; Lively and Jokela 2002). In addition, parasite infection is associated with fluctuations in host genotypes over time (Dybdahl and Lively 1998), and parasites tend to be locally adapted, with higher infectivity on common host genotypes (Lively 1989; Lively and Dybdahl 2000; Dybdahl and Krist 2004; Lively et al. 2004).

In this article, we describe three experiments that compared the fitnesses of locally adapted parasite populations with those of their $\mathrm{F}_{1}$ hybrids. If parasite infection is based on nonadditive gene effects, then $F_{1}$ hybrid parasites should exhibit reduced infectivity (outbreeding depression) compared with the parental average on sympatric hosts, where the reduced infectivity results from the disruption of adaptive combinations of alleles within or between loci. In contrast to this prediction for sympatric hosts, outbreeding depression should be negligible in hosts that are allopatric to both parental parasite populations because allele combinations that are successful in allopatric hosts should be rare in the parental populations. On the other hand, outbreeding depression might be observed for both sympatric and allopatric hosts but only if intrinsic genetic incompatibilities exist between populations (different populations possess alleles that are intrinsically incompatible when they are combined in hybrids). We found that the hybrid fitnesses for parasites were below the parental average but only in comparisons involving sympatric hosts. Thus, our results rule out intrinsic incompatibilities in the genetic basis of population divergence and suggest that local adaptation by these parasites depends on nonadditive gene interactions.

\section{Methods}

\section{Host-Parasite Study System}

Microphallus sp. is a digenetic trematode that inhabits the intestines of waterfowl and wading birds as adults (final hosts) and the gonads of Potamopyrgus antipodarum as larvae (intermediate host), where they ultimately encyst. The infection is passed to the final host when snails infected with cysts (metacercariae) are consumed. In the final host, Microphallus adults excyst in the intestines and undergo sexual reproduction as cross-fertilizing hermaphrodites. Although not formally described, Microphallus sp. displays little genetic differentiation among the South Island populations we studied (Dybdahl and Lively 1996), suggesting that these populations infecting P. antipodarum are members of a single species.

In lab infection experiments, we fed parasite cysts dissected out of infected snails to mice as a final host. The parasites reproduce in the mouse intestinal tract, and parasite eggs are defecated with mouse fecal pellets. Using mice as final hosts, we know that adults produce infective eggs over about $4-5 \mathrm{~d}$. During this period, fecal pellets from each mouse were collected four to six times daily and distributed among treatments and replicate containers. The fecal pellets were washed repeatedly with water to clear away the fecal material. The resulting slurry contained parasite eggs and was distributed among replicate containers with snails. Infection of the snail begins with the ingestion of an egg, migration of the hatched worm to the gonad, and asexual proliferation of larvae that encyst, thereby sterilizing the snail. Individual snails are typically infected by a single Microphallus genotype in the wild (Dybdahl and Lively 1996).

\section{Parasite Population Hybridization and Cross-Infection Experiments}

Experimental infections were carried out in the laboratory at the Edward Percival Field Station, Kaikoura, New Zealand. For each of three experiments, we chose two host populations to serve as sources of the parasite populations from lakes on the South Island of New Zealand. We created $\mathrm{F}_{1}$-generation hybrid populations by feeding an equal dosage of the two parasite populations to each of several mice. If we assume random mating within each mouse, this hybrid population should consist of $50 \% \mathrm{~F}_{1}$ hybrid genotypes and 50\% parental genotypes (25\% from each population). Our statistical analysis provided a test that allowed us to reject assortative mating and selfing for each experiment (see "Statistical Analysis" and "Results"). Random mating is expected because trematodes with life cycles similar to that of Microphallus typically outcross (Criscione and Blouin 2006). In addition, Microphallus shows very little genetic structure or heterozygote deficiency at neutral marker loci (Dybdahl and Lively 1996), suggesting the absence of inbreeding or mating barriers among populations.

A depiction of the general experimental design and details of each experiment are shown in figure A1 and table A1, respectively, in the online edition of the American Naturalist. In general, we performed three reciprocal crossinfection experiments by using parental parasite populations, two host populations that were sympatric to one or the other of the two parental parasite populations, and a hybrid parasite population. In experiments 2 and 3, we also used the parasite populations to expose a host population that was allopatric to both parental hosts (see fig. 
A1). Also in experiments 2 and 3, we created mixed parasite populations, which were mixtures of parasite eggs from the parental parasite populations, to control for the effects of nonrandom mating and to estimate the fitness of $\mathrm{F}_{1}$ hybrid genotypes.

Experiment 1 (Poerua-Ianthe, 1997). We first reanalyze results from a reciprocal cross-infection experiment performed in 1997 between the parasite and snails from Lakes Poerua and Ianthe (Lively and Dybdahl 2000). We created the hybrid population by feeding cysts from 10 infected Poerua snails and 10 infected Ianthe snails to each of four mice. Details of the methods (see Lively and Dybdahl 2000) are similar to those described below, except that the fecal pellets from the four mice were combined before being distributed to the snails to minimize the effect of any individual mouse. Here, we analyze the fitness (prevalence of infection) of the hybrid parasites when exposed to host populations that were sympatric to one of the two parental parasite populations; there was no allopatric host in this experiment.

Experiment 2 (Alexandrina-Poerua, 2001). In this experiment, we chose parental parasite populations from Lakes Alexandrina and Poerua. We used the sympatric snails from Lakes Alexandrina and Poerua and a snail population allopatric to both parental parasites from Lake Ianthe (table A1). We created three mouse replicates for each of the three parasite populations (Alexandrina, Poerua, and the hybrid population). Each mouse replicate of the parasite populations was created according to the details provided in table A1.

For this experiment, we developed a fourth parasite treatment, referred to as the mixed half-dose treatment, in order to estimate the fitness of $F_{1}$ hybrid genotypes within the hybrid population. The hybrid population is expected to be composed of a $50 \%$ dose of $\mathrm{F}_{1}$ hybrid parasite eggs and a $50 \%$ combined dose of eggs from the two parental parasites. To estimate the fitness of a population of purely $\mathrm{F}_{1}$ hybrid genotypes, the mixed half-dose treatment was a $50: 50$ mixture of the two parental parasite populations at half the dose of the hybrid (for detailed methods, see table A2 in the online edition of the American Naturalist).

We used the four parasite populations (parental Alexandrina and Poerua parasites and the hybrid and mixed half-dose parasites) to expose the three host populations (Alexandrina, Poerua, and Ianthe; see fig. A1). We assembled replicate containers of 100 subadult snails for each host population that were size-matched to the smallest subadult snail size in this experiment: selected snails were those that passed through a $1.5-\mathrm{mm}$ sieve but not through a 1.2-mm sieve. To initiate infections, samples from the mouse fecal pellet collections from each mouse replicate were added to three replicate containers. Snails were housed with the parasite eggs for 19 days (January 19February 8). Containers held $2 \mathrm{~L}$ of water, and water was changed twice per day. We also assembled four replicate no-parasite control containers for each lake, with 100 snails to estimate background infection rates for wildcaught snails. The snails were then transported to the United States, where they were held in $2 \mathrm{~L}$ of water with regular water and food changes. After about 107 days from initial exposure to parasite eggs, we dissected the snails from each replicate and recorded their infection status. We subtracted the mean infection rate from the controls for each host from the observed infection rate of each replicate for that host to obtain the experimental infection rate for analysis. One of the three mice that were given parasites from Lake Alexandrina yielded no infections in any of the host populations; because this indicates that the parasite did not reproduce in the mouse, the experimental units that were exposed with this mouse replicate were dropped from all analyses.

Experiment 3 (Alexandrina-Mapourika, 2007). Here, we chose parental parasite populations from Lakes Alexandrina and Mapourika. As an allopatric host population, we chose snails from Lake Paringa. We created two mouse replicates for each of the three parasite populations (parental populations from Alexandrina and Mapourika and the hybrid population) as described in table A1.

In this experiment, we set up a mixed full-dose treatment (see table A2). The goal was to provide a comparison of the fitnesses of $\mathrm{F}_{1}$ hybrid genotypes with those of parental genotypes and to test whether parasites selfed or mated assortatively. Consequently, we set up a mixed fulldose treatment to mimic a full-dose, 50:50 mixture of Alexandrina and Mapourika parasite eggs. To create this treatment, we combined Alexandrina and Mapourika parasite eggs at a dose equal to that of the parental and hybrid treatments (for detailed methods, see table A2).

Again, we performed a laboratory infection experiment using the four parasite populations (Alexandrina, Mapourika, hybrid, and mixed full dose) to expose Alexandrina, Mapourika, and Paringa host populations. To expose snails, we added the eggs from each mouse line to three replicate containers with 50 field-collected snails from each of the three lakes. Subadult snails used for the experiment were those that went through a $2.0-\mathrm{mm}$ sieve but not through a $1.4-\mathrm{mm}$ sieve. Snails were housed with the parasite eggs for 10 days (January 31-February 9) in containers with $2 \mathrm{~L}$ of water. The experimental snails were then transported to the United States, where they were held in $2 \mathrm{~L}$ of water, with regular water and food changes. After 90-100 days from initial exposure to parasite eggs, 
we dissected the snails from each replicate and recorded their infection status. We excluded any infections where the parasite had reached the most advanced developmental stage in order to remove background infections from the analysis (see also Lively and Dybdahl 2000).

\section{Statistical Analysis}

Local Adaptation. Analyses of local adaptation for experiment 1 (Poerua-Ianthe 1997) are described by Lively and Dybdahl (2000). For experiments 2 and 3 (AlexandrinaPoerua 2001 and Alexandrina-Mapourika 2007), we used a mixed-model ANOVA to test for the effect of host population, parasite population, and mouse on observed prevalences; mouse was a random factor nested within parasite population. To satisfy homogeneity of variances and normality assumptions, we calculated the $\arcsin (x)^{1 / 2}$ transformation of prevalences for statistical significance tests. To test for local adaptation of parental parasite populations to their sympatric hosts, we examined the host-parasite interaction term and used pairwise contrasts available in the GLM command of SPSS 11 for Macintosh. These independent contrasts were based on whether the difference between estimated marginal means of the prevalences was nonzero (EMMEANS subcommand). For experiments 2 and 3, we also examined the pairwise contrasts to determine the difference between hybrid and mixed treatments (see tables A3, A4 in the online edition of the American Naturalist).

Hybrid Population Fitnesses. We used the statistical test developed by Hines et al. (2004) to determine whether the fitness of an intermediate form departs from collinear (the additive expectation) compared with the fitnesses of two divergent populations. The test was designed for reciprocal transplant experiments conducted under native environmental conditions when intermediate forms were included, such as interpopulation hybrids. In our experiments, hosts that were sympatric to one or the other of the parental parasite populations are considered the native conditions.

The test for the significance of departure from collinearity is based on a likelihood ratio test under the null hypothesis that the distributions of the fitnesses of the parental and intermediate forms in two environments are collinear. First, estimates of the fitnesses of the three forms in each environment are generated under the collinear null hypothesis, based on observed values and their variances (see Hines et al. 2004, their eq. [B1]). A line that is essentially a best fit of the estimated fitnesses describes an estimated collinear fitness set for intermediate forms. The test estimates $\gamma$, defined as the position of the intermediate form along the fitness set connecting the two parental populations, and then calculates the expectation for the perpendicular departure of the intermediate form from this position. The test statistic reflects the size of the squared differences between observed population fitness means and the estimated population fitness means under the null hypothesis of collinearity for all three forms (two parentals and one intermediate) in two environments (see Hines et al. 2004, their eq. [2]). Hence, the analysis determines the magnitude and significance of the departure of the hybrid parasites from collinearity.

We expect hybrid fitnesses to depart from the collinear expectation in sympatric hosts under several scenarios (fig. 1). If parasite infection is based on nonadditive gene effects (e.g., underdominance or epistasis), then we expect that fitnesses of $F_{1}$ hybrid parasites will exhibit a negative departure from collinearity (outbreeding depression) on the two sympatric hosts. If this outbreeding depression is based on the buildup of specific genetic combinations resulting from host-imposed selection, then we do not expect hybrid fitnesses measured in allopatric hosts to depart from the parental average because adaptive gene combinations for the allopatric host should be rare in the parental parasite populations.

There are several other genetic explanations for outbreeding depression (Lynch and Walsh 1998; Fritz et al. 2003) that we can eliminate using comparisons involving allopatric hosts (fig. 1). If outbreeding depression is caused by intrinsic genetic incompatibilities, the hybridization of allelic combinations between two divergent populations will cause a negative departure from collinearity in sympatric and allopatric host populations alike (see also Hatfield and Schluter 1999). If outbreeding depression is caused by parasite genotype-by-host environment interactions (e.g., hybrid fitness is low in a novel host environment), then we also expect outbreeding depression in comparisons involving allopatric hosts because they represent a novel environment for the parental parasites.

Alternatively, collinear hybrid fitnesses are expected under several scenarios. If parasite infection in the two parasite populations is based on additive gene effects, then we expect the fitnesses of the hybrid population to be near the average of those of the two parental populations. If parasite infection is based on a single locus with dominant gene effects (i.e., dominance of alleles from one parasite population), then the fitnesses of the hybrid population should be similar to that of one of the parental populations, and the fitnesses of the hybrid population should be collinear and close to that of the parental population that possesses the dominant alleles. Finally, if the experimental hybrid population contains no $\mathrm{F}_{1}$ hybrid genotypes, due to assortative mating or selfing, but only a mixture of pure genotypes, then the fitness pair of the hybrid population should be collinear. 


\begin{tabular}{|c|c|c|c|}
\hline Possible & e outcomes & & Genetic inference \\
\hline & Host population & $\begin{array}{l}\text { Deviation from } \\
\text { collinearity }\end{array}$ & \multirow{3}{*}{$\begin{array}{l}\text { Nonadditive genetic combinations under } \\
\text { host-driven selection }\end{array}$} \\
\hline \multicolumn{4}{|l|}{$\begin{array}{l}\text { Hybrid fitnesses below parental } \\
\text { average (outbreeding depression) } \\
\text { in sympatric hosts }\end{array}$} \\
\hline & Sympatric & - & \\
\hline & Allopatric & 0 & \\
\hline & Sympatric & - & \\
\hline & Allopatric & - & Intrinsic genetic incompatibilities \\
\hline & Sympatric & - & Negative parasite genotype-host \\
\hline & Allopatric & - & environment (nongenetic) effects \\
\hline \multicolumn{4}{|l|}{$\begin{array}{l}\text { Hybrid fitnesses not different from } \\
\text { parental average in sympatric } \\
\text { hosts }\end{array}$} \\
\hline & Sympatric & 0 & \multirow{2}{*}{$\begin{array}{l}\text { Additive or single-locus dominance } \\
\text { effects }\end{array}$} \\
\hline & Allopatric & 0 & \\
\hline & Sympatric & 0 & \multirow{2}{*}{$\begin{array}{l}\text { Assortative mating by population or } \\
\text { selfing, hybrids not formed }\end{array}$} \\
\hline & Allopatric & 0 & \\
\hline
\end{tabular}

Figure 1: Possible outcomes and inferences for the comparisons of the fitness of interpopulation hybrid parasites versus their parental populations. Fitness is measured in hosts that are sympatric to one or the other of the parental parasite populations (representing native conditions for the divergent populations) versus hosts that are allopatric to both parental parasites. Deviations from collinearity are determined by the Hines et al. (2004) analysis. A zero indicates no significant deviation from collinearity; a minus sign indicates a significant negative deviation.

In summary, nonadditive gene effects and tight hostdriven selection are supported if the hybrid fitness set is below the line for the comparisons between sympatric hosts only (see fig. 1). Our test for outbreeding depression using the hybrid population is conservative to departures from random mating: estimates of hybrid fitnesses will be drawn closer to the line (collinear) by the parental genotypes formed from random mating or from selfing or assortative mating.

We calculated the test statistic $L$, which reflects the (squared) size of the displacement of the intermediate fitnesses to one side or the other of the straight line joining the fitnesses of the two divergent populations; $L$ has an asymptotic $\chi^{2}$ distribution with $1 \mathrm{df}$. The test is robust and statistically powerful and does not rely on normally distributed data or equal variances (Hines et al. 2004). Fitnesses (prevalences) were the corrected marginal means and their standard deviations from the ANOVA model using untransformed data.

To display the analysis results, we plotted the observed prevalence values for each parasite population and the prevalences estimated by the Hines et al. (2004) analysis. If the observed hybrid fitnesses are collinear with the fitnesses of divergent forms (null hypothesis), then $L$ will be close to 0 . When $L$ is statistically different from 0 , then the null hypothesis of collinearity can be rejected. If $L$ is statistically different from 0 for any of the hosts, we conclude that nonrandom mating was unimportant because the same parasite lines were used across all hosts. We performed one-tailed tests of the hypothesis that hybrid fitnesses would be below the collinear fitness set, as anticipated by the disruption of nonadditive gene combinations and tight host specificity.

Fitness of $F_{1}$ Hybrid Genotypes. For experiment 2 (Alexandrina-Poerua 2001), we also estimated the fitness of a population consisting of only $\mathrm{F}_{1}$ hybrid genotypes, using the mixed half-dose and hybrid treatments. The mixed half-dose treatment mimicked the expected dose and ratio of the parental genotypes in the hybrid treatment. Hence, the prevalence of $F_{1}$ genotypes was calculated as twice the mean difference between the mixed half-dose and hybrid treatments. This estimate depends on a linear doseresponse relationship, so we used the same ANOVA described earlier but with $-\ln (1-x)$-transformed prevalences. This transformation linearizes the diminishing returns in the dose-response relationship (Osnas and Lively 2004). We plotted the estimated prevalence of $F_{1}$ hybrid genotypes along with the prevalences of the parental genotypes and their confidence intervals. (Because the homogeneity of variances assumption was violated for the 
$-\ln (1-x)$-transformed data, we did not use the $P$ values for significance tests from this analysis.)

\section{Results \\ Local Adaptation}

For all three experiments, both parental parasite populations were locally adapted, as indicated by the significant host-parasite interaction (table 1; for the analysis of experiment 1 , see Lively and Dybdahl 2000). Pairwise contrasts showed that each parental parasite population was more infective to its sympatric host (fig. 2; tables A3, A4). In experiment 2, Alexandrina parasites were significantly more infective to Lake Alexandrina hosts than they were to Lake Poerua and Ianthe hosts $(P<.001$ for both), and they were more infective to Lake Poerua hosts than to Lake Ianthe hosts $(P<.001)$. Poerua parasites were significantly more infective to Poerua hosts than were Alexandrina and Ianthe parasites $(P<.001$ for both). Poerua parasites were also more infective to Alexandrina hosts than to Ianthe hosts $(P<.012)$. In experiment 3 , pairwise contrasts indicated that Lake Alexandrina parasites were significantly more infective to Lake Alexandrina hosts than to Mapourika and Paringa hosts $(P<.001$ for both). Infectivity of Alexandrina parasites to Mapourika and Paringa hosts did not differ significantly $(P=.738)$. Mapourika parasites were more infective to Mapourika hosts than to Alexandrina and Paringa hosts $(P<.005$ for both). Infectivity of Mapourika parasites to Alexandrina and $\mathrm{Pa}$ ringa hosts also differed significantly $(P=.045)$.

\section{Hybrid Population Fitnesses}

For all three experiments, the analysis of collinearity for sympatric hosts showed a consistent pattern of significant negative deviations of hybrid fitnesses compared with the fitnesses of the two parental populations (table 2; fig. 2). In experiment 1 , hybrid fitness was significantly noncollinear $(P<.0001)$, and the departure was negative (fig. $2 a)$. The negative deviation from collinearity was also significant in experiment 2 for sympatric hosts $(P=.041$; fig. $2 b$ ). However, in experiment 2, hybrid fitnesses did not deviate significantly from collinearity when the comparison involved allopatric Lake Ianthe hosts $(P=.248$ with Alexandrina hosts and $P=.367$ with Poerua hosts; table 2; fig. $3 a, 3 b)$.

In experiment 2 , the hybrid parasite treatment was significantly more infective than the mixed half-dose treatment in the allopatric host (Ianthe), according to the pairwise contrast $(P=.029)$, but was either marginally or not significantly more infective in the sympatric hosts $(P=$ .050 for Alexandrina hosts and $P=.183$ for Poerua hosts;
Table 1: Mixed-model ANOVA of infection prevalences

\begin{tabular}{|c|c|c|c|c|}
\hline Experiment and source & $\mathrm{df}$ & MS & $F$ & $P$ \\
\hline \multicolumn{5}{|l|}{ 2. Alexandrina-Poerua 2001: } \\
\hline Host & 2 & 1.454 & $42.378^{\mathrm{B}}$ & $<.001$ \\
\hline Parasite & 3 & .265 & $3.264^{\mathrm{A}}$ & .101 \\
\hline Host $\times$ parasite & 6 & .196 & $5.713^{\mathrm{B}}$ & .005 \\
\hline Mouse $(\text { parasite })^{\mathrm{A}}$ & 6 & .0812 & $2.366^{\mathrm{B}}$ & .096 \\
\hline Host $\times$ mouse $(\text { parasite })^{\mathrm{B}}$ & 12 & .0343 & $3.431^{\mathrm{C}}$ & .001 \\
\hline Error $^{\mathrm{C}}$ & 60 & .0105 & & \\
\hline \multicolumn{5}{|c|}{ 3. Alexandrina-Mapourika 2007: } \\
\hline Host & 2 & .232 & $17.541^{\mathrm{B}}$ & .001 \\
\hline Parasite & 3 & .022 & $.485^{\mathrm{A}}$ & .711 \\
\hline Host $\times$ parasite & 6 & .123 & $9.274^{\mathrm{B}}$ & .003 \\
\hline Mouse $(\text { parasite })^{\mathrm{A}}$ & 4 & .045 & $3.437^{\mathrm{B}}$ & .065 \\
\hline Host $\times$ mouse $(\text { parasite })^{\mathrm{B}}$ & 8 & .013 & $2.034^{\mathrm{C}}$ & .062 \\
\hline Error $^{\mathrm{C}}$ & 48 & .0065 & & \\
\hline
\end{tabular}

Note: Differential fitnesses of parasite populations to different host populations are indicated by the significant host $\times$ parasite interaction. Prevalences were $\arcsin (x)^{1 / 2}$ transformed. Mouse was designated as a random effect and nested within parasite. Superscripts indicate the source error term for each $F$-test. Boldface indicates $P$ values $<.05$. MS $=$ mean square.

see also table A3). Hence, $F_{1}$ hybrid genotypes contributed to fitness in the allopatric host, consistent with the absence of intrinsic genetic incompatibilities.

The negative deviation from collinearity for hybrid fitnesses in sympatric hosts was also significant in experiment $3(P=.028$; fig. $2 c)$. Again, the hybrid fitnesses did not deviate significantly from collinearity when the comparison involved allopatric Lake Paringa hosts (table 2; fig. $3 c$, $3 d$ ). As in experiment 2, there is no evidence that the hybrids had above- or below-average fitness compared with that of the parental species when measured in allopatric hosts.

In experiment 3 , we compared the fitnesses of the hybrid population with the fitnesses of the mixed full-dose parasites in the sympatric hosts. According to the Hines et al. (2004) analysis, the hybrid fitnesses were significantly lower than the collinear expectation $(P=.028)$, but the mixed full-dose fitnesses were slightly higher than the collinear expectation $(P=.011)$, suggesting that hybrid genotypes were less fit than parental genotypes and that parasites did not self or mate assortatively (fig. $2 c$ ).

\section{Fitness of $F_{1}$ Hybrid Genotypes}

We calculated the prevalences of $F_{1}$ hybrid genotypes based on our hybrid and mixed half-dose parasites for experiment 2 (Alexandrina-Poerua 2001). Consistent with our tests of collinearity, the estimated prevalences of hybrid genotypes for both Lake Alexandrina and Lake Poerua hosts were below the intermediate phenotype (see confidence intervals in fig. $4 a, 4 b$ ). In contrast, for the allopatric 
a.

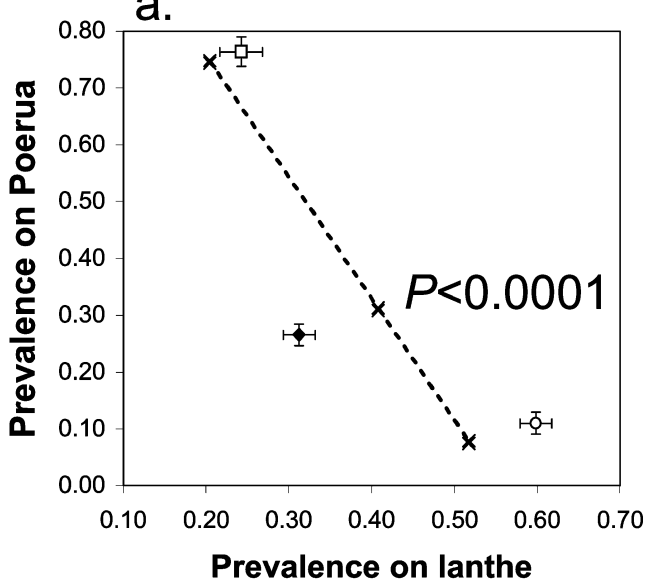

C.

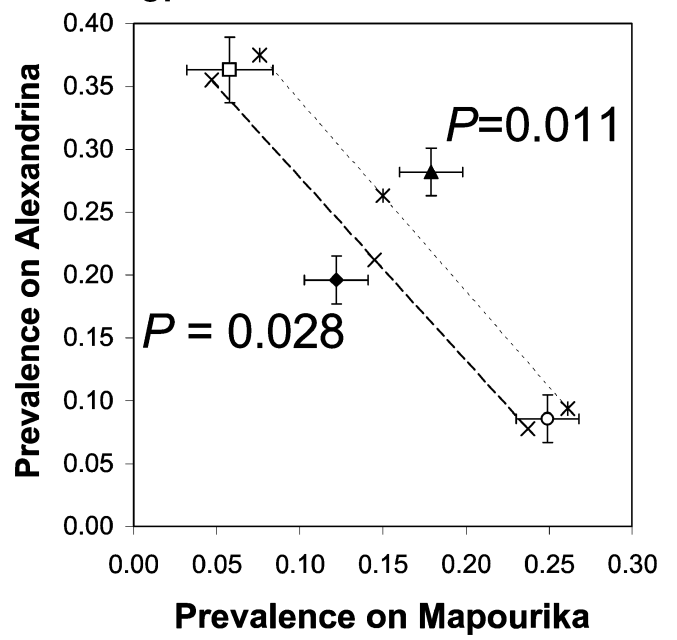

b.

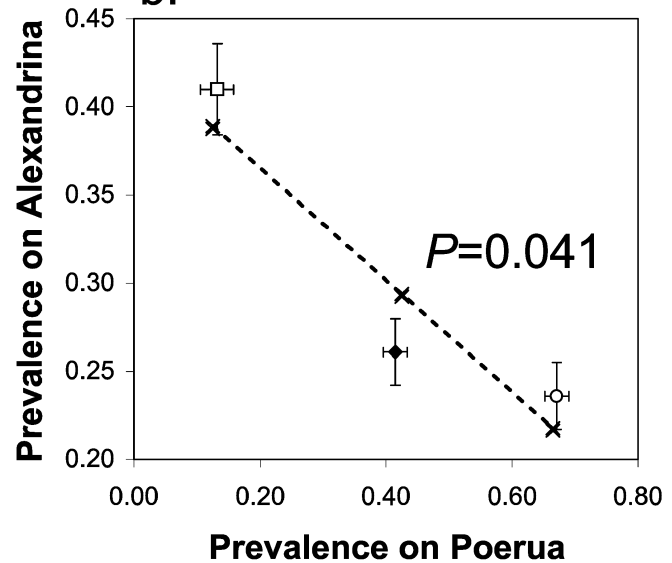

Figure 2: Fitnesses of hybrid parasite populations compared with those of parental populations in sympatric hosts for the three infection experiments. Significant $P$ values indicate departures from collinearity for the hybrid population in all experiments and a departure from collinearity for the mixed full-dose population in experiment 3 (Alexandrina-Mapourika 2007). Open symbols = parental parasite; filled diamond = hybrid parasites; cross $=$ estimated means from the Hines et al. (2004, their eq. [B1]) analysis for the tests of collinearity of hybrids. The trendline connects estimated means. $a$, Experiment 1 (Poerua-Ianthe 2001). Open square $=$ Poerua parasites; open circle $=$ Ianthe parasites. $b$, Experiment 2 (Alexandrina-Poerua 2001). Open square $=$ Alexandrina parasites; open circle $=$ Poerua parasites. $c$, Experiment 3 (Alexandrina-Mapourika 2007). Open square $=$ Alexandrina parasites; open circle = Mapourika parasites; filled triangle $=$ mixed full-dose parasites; cross with line $=$ estimated means for the test of collinearity of mixed full dose; bold dashed line $=$ trendline estimate for hybrids; light dashed line $=$ trendline for mixed full-dose treatment.

Lake Ianthe hosts, the infectivity of $F_{1}$ hybrid genotypes was relatively higher and not distinguishable from the that of intermediate phenotype (fig. $4 c$ ).

\section{Discussion}

We studied the relative fitness of interpopulation hybrids between locally adapted parasite populations in order to better understand the genetic basis for parasite infection. In three different experiments, we found that hybrid genotypes exhibited outbreeding depression on sympatric host populations (fig. 2; fig. $4 a, 4 b$ ). In contrast, hybrid fitnesses did not depart significantly from those of the average phenotype of parental populations in allopatric host populations (Ianthe in experiment 2 and Paringa in experiment 3; figs. 3, 4c). Moreover, the fitness of the hybrid population was significantly higher than that of the mixed half-dose treatment (a half-dose mixture of parental parasites) in the allopatric host (Ianthe host in experiment 2 ). These two sets of results with allopatric hosts are inconsistent with the hypothesis that the outbreeding depression observed in hybrids on sympatric host popula- 
Table 2: Analysis of collinearity of fitnesses of interpopulation hybrid parasites or mixed full-dose parasites with their parental populations in pairs of hosts

\begin{tabular}{|c|c|c|c|c|c|c|c|}
\hline Host 1 & Host 2 & Parasite & Host 2 source & $\gamma$ & $L$ & $P$ & Deviation \\
\hline \multicolumn{8}{|c|}{ Experiment 1 (Poerua-Ianthe 1997): } \\
\hline Poerua & Ianthe & Hybrid & Sympatric & .350 & 16.273 & $<.0001$ & - \\
\hline \multicolumn{8}{|c|}{ Experiment 2 (Alexandrina-Poerua 2001): } \\
\hline Alexandrina & Poerua & Hybrid & Sympatric & .445 & 3.042 & .041 & - \\
\hline Alexandrina & Ianthe & Hybrid & Allopatric & .200 & .464 & .248 & 0 \\
\hline Poerua & Ianthe & Hybrid & Allopatric & .471 & .115 & .367 & 0 \\
\hline \multicolumn{8}{|c|}{ Experiment 3 (Alexandrina-Mapourika 2007): } \\
\hline Alexandrina & Mapourika & Hybrid & Sympatric & .483 & 3.648 & .028 & - \\
\hline Alexandrina & Paringa & Hybrid & Allopatric & .436 & 2.268 & .066 & 0 \\
\hline Mapourika & Paringa & Hybrid & Allopatric & .311 & .187 & .332 & 0 \\
\hline Alexandrina & Mapourika & Mixed full dose & Sympatric & .601 & 5.243 & .011 & + \\
\hline
\end{tabular}

Note: Host 2 was either sympatric or allopatric to both divergent parasites. The analysis is repeated for three similar experiments. Bold values are statistically significant in a one-tailed test for negative deviations from collinearity. For deviations, 0 indicates no significant deviations from collinearity, a minus sign indicates significant negative deviations, and plus sign indicates significant positive deviations. For all tests, $\mathrm{df}=1 . L=$ test statistic reflecting the (squared) size of the displacement of the intermediate fitnesses to one side or the other of the straight line joining the fitnesses of the two divergent populations.

tions was caused by intrinsic (e.g., physiological) genetic factors that had diverged between the parental populations. Taken together, the results indicate that hybridization between divergent parasite populations disrupts adaptive gene combinations within or between loci that had resulted from host-imposed selection.

In general, a comparison of fitnesses of $\mathrm{F}_{1}$ hybrids relative to those of the average phenotype of parental populations is useful for determining the underlying genetic basis of a trait (Lynch and Walsh 1998; Fritz et al. 1999, 2003). The pattern of consistent outbreeding depression on sympatric hosts strongly suggests that the genetic basis of infection must be nonadditive (fig. 1). Because the hybrid population was significantly noncollinear with the fitness set joining parental phenotypes, the additive model of gene action used in some coevolutionary theories (Gavrilets 1997; Ridenhour and Nuismer 2007) can be rejected for our system. In addition, single-locus dominant gene action can also be rejected because the hybrids did not consistently resemble one or the other of the parental populations in the sympatric hosts (fig. 2; fig. 4a, 4b).

Outbreeding depression in the $\mathrm{F}_{1}$ generation might implicate negative parasite genotype-by-host environment interactions. For example, the depressed fitnesses of the hybrid genotype might be governed by dominance of alleles from one parasite population in the novel host environment (e.g., for experiment 3, dominance of the $\mathrm{Al}$ exandrina alleles in the novel Mapourika host environment and dominance of the Mapourika alleles in the novel Alexandrina host environment), leading to low hybrid fitness in both. However, host environment should be largely determined by host genotype, especially because environmental variation is minimized in our laboratory experimental infections. Hence, the fitnesses we measured in the lab infection experiments should be the result of parasite genotype-by-host genotype interactions rather than parasite genotype-by-host environment interactions (Nuismer and Gandon 2008). The assumption of parasite genotype-by-host genotype interactions is strengthened by our results from the allopatric host combinations. If outbreeding depression were influenced by parasite genotypeby-host environment interactions, then one of the parental sets of alleles should have been dominant in the novel allopatric host environment (Ianthe in experiment 2 and Paringa in experiment 3 ), which is contrary to our results (figs. 3, 4c).

Finally, with the absence of support for other sources of outbreeding depression in the $F_{1}$ generation, our results suggest that other nonadditive gene effects such as underdominance or epistasis (Lynch and Walsh 1998; Fritz et al. 1999, 2003) determine parasite infection. Such nonadditive gene effects are implicit in models of host-parasite interactions that underlie important coevolutionary theory, including matching allele, inverse matching allele, gene for gene, or combinations of these (e.g., Agrawal and Lively 2002, 2003; Nuismer and Otto 2004; Otto and Nuismer 2004; Nuismer 2006). Among these models, our results are consistent with the matching allele model, which is expected to describe invertebrate self/nonself defense systems. Under the matching allele model, parasites must express a narrow array of antigens or elicitors that match host recognition sites in order to evade host defenses (reviewed in Otto and Michalakis 1998; Dybdahl and Storfer 2003; Lambrechts et al. 2006). Under such a model, one might expect low hybrid fitness in parasites because hybridization will break up gene combinations that permit evasion of the local host defenses. However, at this point, 
a.

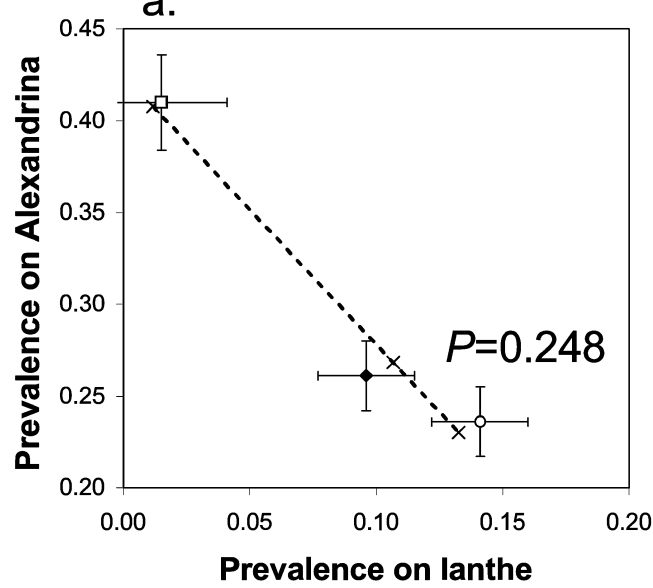

b.

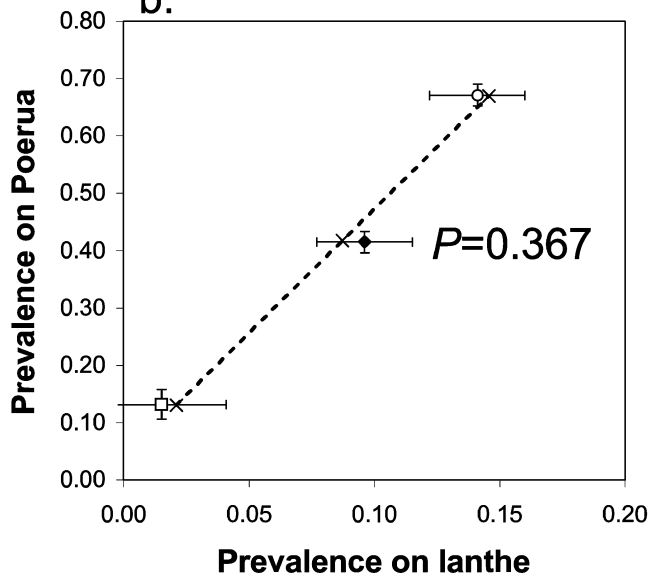

C.

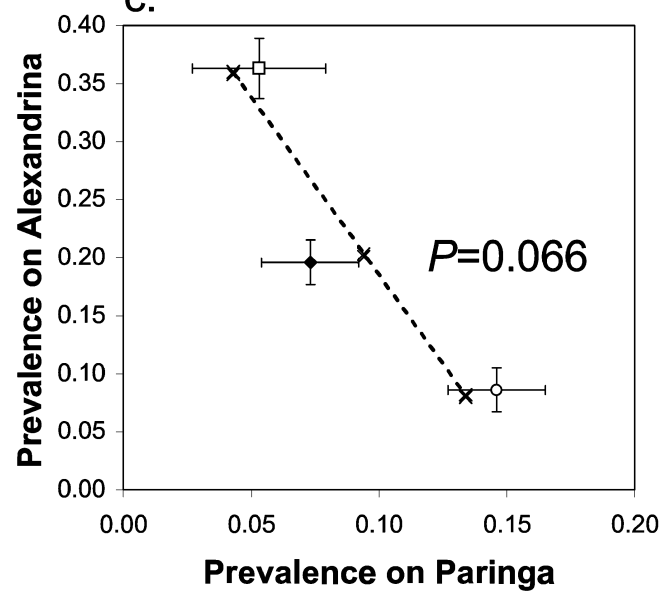

d.

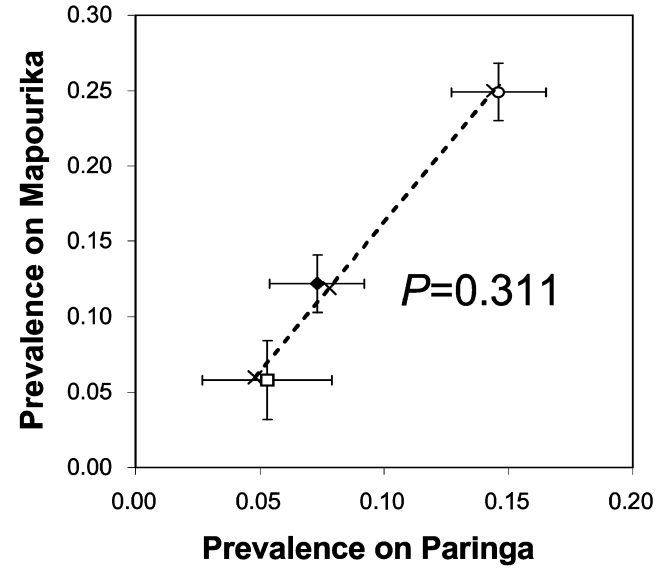

Figure 3: Fitnesses of hybrid parasite populations compared with those of parental parasite populations in the allopatric hosts for two of the infection experiments. Nonsignificant $P$ values indicate that hybrid population fitness was collinear in all comparisons. Open symbols $=$ parental parasite; filled diamond $=$ hybrid parasites; cross $=$ values fitted by the Hines et al. (2004) analysis. The trendlines connect fitted values. $a$, $b$, Experiment 2 (Alexandrina-Poerua 2001). Open squares = Alexandrina parasites; open circles = Poerua parasites. $c$, $d$, Experiment 3 (Alexandrina-Mapourika 2007). Open squares $=$ Alexandrina parasites; open circles $=$ Mapourika parasites.

there is little information available from coevolutionary theory to rule out the alternative models.

The consistent pattern of outbreeding depression in sympatric hosts indicates that parasites did not mate assortatively or self in the mouse. Under assortative mating or selfing, the hybrid population would have been up to $100 \%$ parental genotypes $(50 \%$ of each parental parasite population). If so, the hybrid population fitnesses would have been indistinguishable from the fitnesses connecting the divergent parental populations, in contrast to our results (table 2; fig. 2). In an experimental verification that assortative mating and selfing were not important, we found that the fitnesses of the hybrid population showed a significant negative deviation from collinearity in sympatric hosts but that the mixed full-dose deviation was positive (experiment 3 [Alexandrina-Mapourika 2007]; fig. $2 c$ ). These results were not surprising in light of other tests for inbreeding and assortative mating in trematodes (Dybdahl and Lively 1996; Criscione and Blouin 2006). In any case, our tests were conservative to departures from random mating because any level of assortative mating and selfing would bring the fitnesses of the hybrid population closer to collinear in all host populations.

In conclusion, we examined hybrid fitness in coevolving, locally adapted parasites in an effort to determine the genetic basis of infection and parasite divergence. We found a consistent pattern of outbreeding depression when comparing hybrid fitnesses with the fitnesses of their parental populations in their sympatric hosts but not with those of their allopatric hosts. These results suggest that allelic 
a. Poerua host

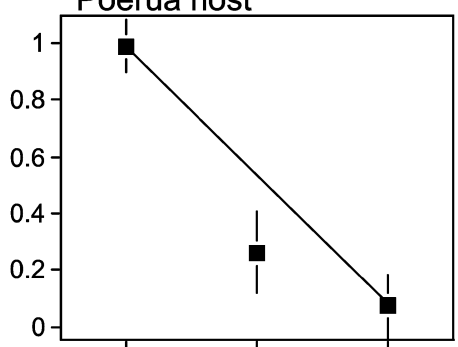

b. Alexandrina host

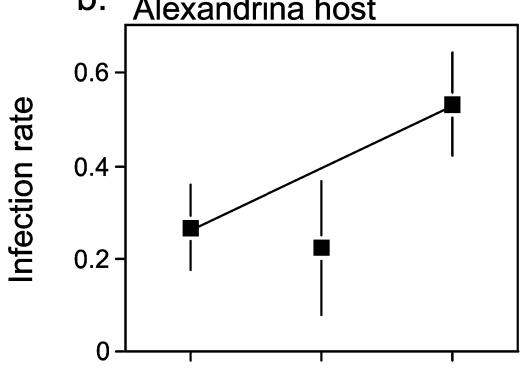

C. lanthe host

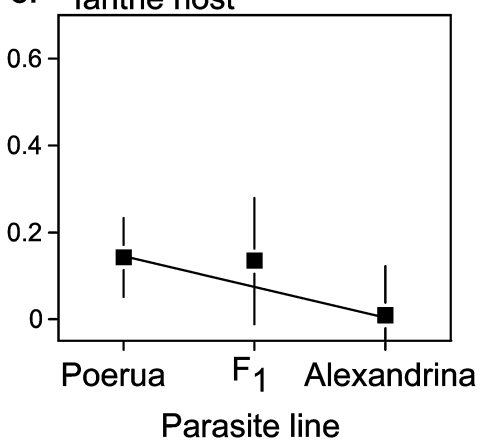

Figure 4: Prevalence of infection in experiment 2 (Alexandrina-Poerua 2001) by parental parasite populations and the estimated prevalence by $\mathrm{F}_{1}$ hybrid genotypes for each host population. Plots show $-\ln (1-x)$ transformed means and 95\% confidence intervals derived from the ANOVA. Prevalences of parental lines are connected to visualize the intermediate phenotype for comparison with $\mathrm{F}_{1}$ prevalences and confidence intervals. The estimated $\mathrm{F}_{1}$ means are significantly below the intermediate phenotype on the sympatric hosts (Poerua and Alexandrina) but do not differ from the intermediate phenotype in the allopatric host (Ianthe), as determined by the confidence intervals.

combinations were nonadditive in interactions with the sympatric hosts for which the parasites were adapted. There was no indication of single-locus dominance, intrinsic genetic incompatibilities, or negative parasite genotype-by-host environment interactions in our results. Hence, the observed outbreeding depression is consistent with other nonadditive forms of gene action (single-locus underdominance, multilocus dominance, or epistasis) and genotype-specific host-driven selection. Given the importance of specific genetic combinations in determining infection, we believe that host-parasite interactions in this system could contribute to selection for sex and recombination in a changing coevolutionary environment, as envisioned by the Red Queen hypothesis.

\section{Acknowledgments}

We thank C. Fenster, S. Nuismer, K. Räsänen, M. Wade, the Ecology, Evolution, and Genetic Reading Group at Washington State University (WSU), and the Coevolvers Reading Group at the University of Idaho and WSU for comments on the manuscript. We thank J. McKenzie, J. van Berkel, and the Edward Percival Field Station staff for invaluable logistical support. This study was supported by the National Science Foundation (grants DEB-9907373 to M.F.D., DEB-9904840 to C.M.L., DEB-0210971 to L.F.D., and DEB-0660639 to J.J. and C.M.L.) and the Swiss National Science Foundation and the Academy of Finland (to J.J.).

\section{Literature Cited}

Agrawal, A. F., and C. M. Lively. 2002. Infection genetics: gene-forgene versus matching-allele models and all points in between. Evolutionary Ecology Research 4:79-90.

- 2003. Modeling infection genetics as a two-step process combining gene-for-gene and matching-allele genetics. Proceedings of the Royal Society B: Biological Sciences 270:323-334.

Carius, H.-J., T. J. Little, and D. Ebert. 2001. Genetic variation in a host-parasite association: potential for coevolution and frequencydependent selection. Evolution 55:1136-1145.

Criscione, C. D., and M. S. Blouin. 2006. Minimal selfing, few clones, and no among-host genetic structure in a hermaphroditic parasite with asexual larval propagation. Evolution 60:553-562.

Dybdahl, M. F., and A. C. Krist. 2004. Genotypic vs. condition effects on parasite-driven rare advantage. Journal of Evolutionary Biology 17:967-973.

Dybdahl, M. F., and C. M. Lively. 1995. Diverse, endemic and polyphyletic clones in mixed populations of a freshwater snail (Potamopyrgus antipodarum). Journal of Evolutionary Biology 8:385389 .

1996. The geography of coevolution: comparative population structures for a snail and its trematode parasite. Evolution 50:2264-2275.

1998. Host-parasite coevolution: evidence for rare advantage and time-lagged selection in a natural population. Evolution 52: 1057-1066.

Dybdahl, M. F., and A. Storfer. 2003. Parasite local adaptation: Red Queen versus Suicide King. Trends in Ecology \& Evolution 18: 523-530.

Fritz, R. S., C. Moulia, and G. Newcombe. 1999. Resistance of hybrid plants and animals to herbivores, pathogens, and parasites. Annual Review of Ecology and Systematics 30:565-591.

Fritz, R. S., C. G. Hochwender, S. J. Brunsfeld, and B. M. Roche. 2003. Genetic architecture of susceptibility to herbivores in hybrid willows. Journal of Evolutionary Biology 16:1115-1126.

Gandon, S., Y. Capowiez, Y. Dubois, Y. Michalakis, and I. Olivieri. 1996. Local adaptation and gene-for-gene coevolution in a meta- 
population model. Proceedings of the Royal Society B: Biological Sciences 263:1003-1009.

Gavrilets, S. 1997. Coevolutionary chase in exploiter-victim systems with polygenic characters. Journal of Theoretical Biology 186:527534.

Hamilton, W. D. 1980. Sex versus non-sex versus parasite. Oikos 35: 282-290.

Hatfield, T., and D. Schluter. 1999. Ecological speciation in sticklebacks: environment-dependent hybrid fitness. Evolution 53:866873.

Hines, R. J., W. G. Hines, and B. W. Robinson. 2004. A new statistical test of fitness set data from reciprocal transplant experiments involving intermediate phenotypes. American Naturalist 163:97-104.

Howard, R. S., and C. M. Lively. 1994. Parasitism, mutation accumulation and the maintenance of sex. Nature 367:554-557.

Jaenike, J. 1978. An hypothesis to account for the maintenance of sex within populations. Evolutionary Theory 3:191-194.

Jokela, J., and C. M. Lively. 1995. Spatial variation for infection by digenetic trematodes in a population of freshwater snails (Potamopyrgus antipodarum). Oecologia (Berlin) 103:509-517.

Lambrechts, L., S. Fellous, and J. C. Koella. 2006. Coevolutionary interactions between host and parasite genotypes. Trends in Parasitology 22:12-16.

Lively, C. M. 1987. Evidence from a New Zealand snail for the maintenance of sex by parasitism. Nature 328:519-521.

1989. Adaptation by a parasitic trematode to local populations of its snail host. Evolution 43:1663-1671.

. 1992. Parthenogenesis in a freshwater snail: reproductive assurance versus parasitic release. Evolution 46:907-913.

1999. Migration, virulence, and the geographic mosaic of adaptation by parasites. American Naturalist 153(suppl.):S34-S47.

Lively, C. M., and M. F. Dybdahl. 2000. Parasite adaptation to locally common host genotypes. Nature 405:679-681.

Lively, C. M., and J. Jokela. 2002. Temporal and spatial distributions of parasites and sex in a freshwater snail. Evolutionary Ecology Research 4:219-226.

Lively, C. M., M. F. Dybdahl, J. Jokela, E. E. Osnas, and L. F. Delph. 2004. Host sex and local adaptation by parasites in a snail-trematode interaction. American Naturalist 164(suppl.):S6-S18.

Lynch, M., and B. Walsh. 1998. Genetics and analysis of quantitative traits. Sinauer, Sunderland, MA.

Nuismer, S. L. 2006. Parasite local adaptation in a geographic mosaic. Evolution 60:83-88.

Nuismer, S. L., and S. Gandon. 2008. Moving beyond common garden and transplant designs: insight into the causes of local adaptation in species interactions. American Naturalist 171:658-668.

Nuismer, S. L., and S. P. Otto. 2004. Host-parasite interactions and the evolution of ploidy. Proceedings of the National Academy of Sciences of the USA 101:11036-11039.

Osnas, E. E., and C. M. Lively. 2004. Parasite dose, prevalence of infection and local adaptation in a host-parasite system. Parasitology 128:223-228.

Otto, S. P., and Y. Michalakis. 1998. The evolution of recombination in changing environments. Trends in Ecology \& Evolution 13:145151.

Otto, S. P., and S. L. Nuismer. 2004. Species interactions and the evolution of sex. Science 304:1018-1020.

Peters, A. D., and C. M. Lively. 1999. The Red Queen and fluctuating epistasis: a population genetic analysis of antagonistic coevolution. American Naturalist 154:393-405.

. 2007. Short- and long-term benefits and detriments to recombination under antagonistic coevolution. Journal of Evolutionary Biology 20:1206-1217.

Ridenhour, B. J., and S. L. Nuismer. 2007. Polygenic traits and parasite local adaptation. Evolution 61:368-376.

Associate Editor: Yannis Michalakis Editor: Michael C. Whitlock 